\title{
The Effect of Nd:YAG Laser and \\ Casein- Phosphor-Peptide-Amorphous Calcium Phosphate with Fluoride on Dental Enamel De-mineralization: In vitro study
}

\author{
Hiba Issam Abdulahhab \\ M.Sc, B.D.S \\ Ali Shukur Mahmood \\ Assist Proff CABS,MBCh,HDIPLM
}

\begin{abstract}
The aim of the study was to evaluate a new treatment modality to decrease dental enamel demineralization via combing the effect of pulsed Neodymium Yttrium Garnet (Nd:YAG) laser (1064 nm) and Re-mineralizing paste(CPP-ACP containing sodium fluoride) regarding the tooth pulp chamber temperature elevation, calcium concentration dissolute from enamel surfaces and the dental enamel absorption of methylene blue stain by assessing ( $R$ red , $G$ green ,B blue)values. Sixty human permanent premolars were used for measuring calcium concentration dissolution and $(R, G, B) s$ values. They randomly divided into six groups: the negative control group (A) received no treatment, and the positive control group (B) where the re-mineralizing paste was applied without laser irradiation. For the groups (C), (D), (E) and (F) the $(1064 \mathrm{~nm})$ pulsed Nd:YAG laser was used in two dose settings. The lower laser dose setting at $(60 \mathrm{~mJ})$ for the $C$ and $D$ groups,and the higher laser dose setting at (170mJ)for $E$ and $F$ groups respectively. Groups $D$ and $E$ in addition to laser irradiation the Re-mineralizing paste was applied.

The atomic absorption photometry test results showed that groups $C$, and D were statistically different compared to the groups $A$ and $B(p<0.001)$ in the reduction of calcium dissolution, Whereas Groups $E$ and $F$ shown non-significant reduction in calcium dissolution compared to control groups.

Twenty teeth were used to assess the temperature elevation during irradiation using a K-type thermocouple at $37 \pm 0.5^{\circ} \mathrm{C}$, divided for ten teeth for each irradiation settings. The maximum tempers of temperature test showed that the maximum temperature increases for group $E$ was very high significant difference $(p<0.05)$ over that of groups $C$.

The $(R, G, B) s$ value test showed that groups $C$ and $D$ having lower affinity to absorb methylene blue stain, and they are statically significant different compared to the groups $A$ and $B$.while groups $E$ and $F$ showing a higher affinity to absorb methylene blue stain compared to control groups.
\end{abstract}

\section{KEYWORDS}

pulsed $\mathrm{Nd}$ :YAG laser ,(R,G,B) values, enamel demineralization.

CITE THIS ARTCLE

Abdulahhab H, Mahmood A. The Effect of Nd:YAG Laser and Casein- Phosphor-Peptide-Amorphous Calcium Phosphate with Fluoride on Dental Enamel De-mineralization: In vitro study. Iraqi Dent. J. 2015; 37(3):129-133. http://www.iraqidentaljournal.com

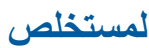

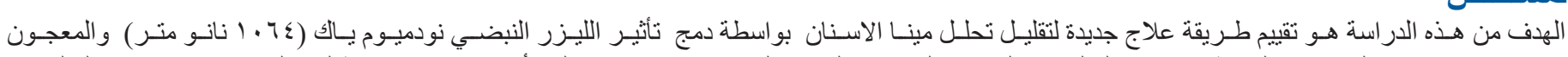

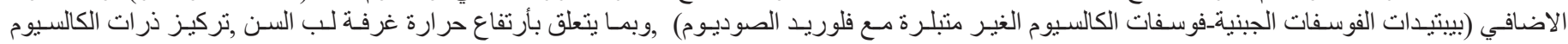

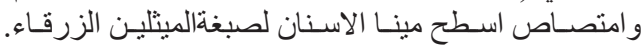

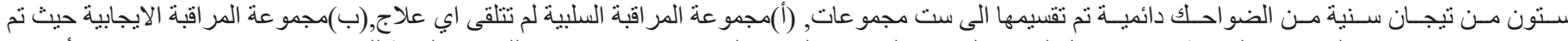

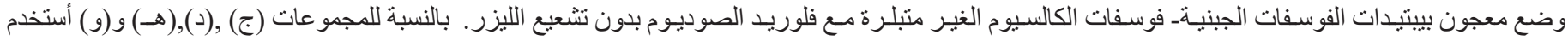

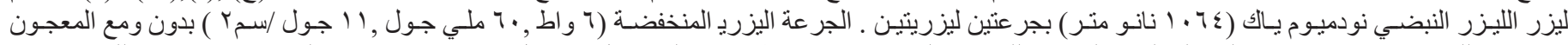

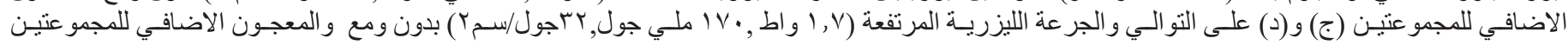

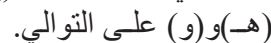

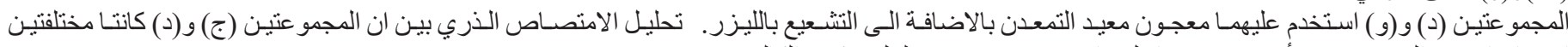

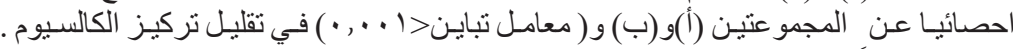

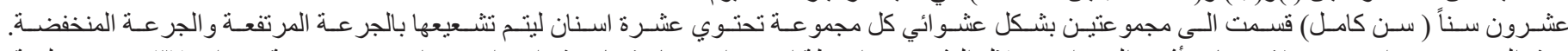

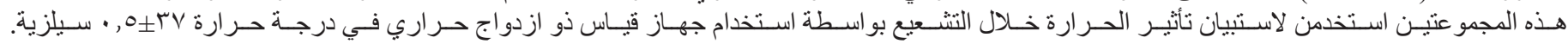

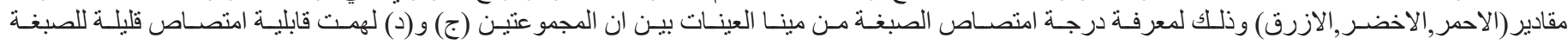

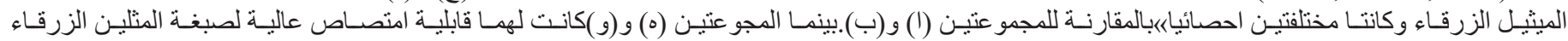

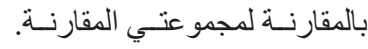

\section{INTRODUCTION}

Dental caries is continuous most prevalent disease during childhood and Adolescence ${ }^{(1)}$. Dairy products including milk, milk concentrates and cheese have long been known to exhibit anti-caries activity where the responsible components being identified as casein, calcium and phosphate. The repair of early tooth enamel lesions has been recently demonstrated by tryptic phospho-peptides derived from milk caseins that associate with amorphous calcium phosphate forming stable complexes ${ }^{(2)}$. In addition to fluoride, a casein phosphopeptide- amorphous calcium phosphate (CPP-ACP) and fluoride containing CPPACP pastes have also been recommended for caries 
prevention and enamel remineralization ${ }^{(3,4,5)}$. Since the 1960s, it has been consistently confirmed that lasers can significantly increase the acid resistance of enamel, and when associated with fluoride, a significant synergism between them as been shown in the reduction of enamel solubility $\left.{ }^{(}\right)$.

The use of lasers in caries prevention was first suggested in 1972 using a ruby laser, since then, many investigations related to the application of other lasers, such as argon, $\mathrm{CO}_{2}$, and Nd:YAG lasers, in the area of preventive dentistry, have been carried out and laser irradiation has been proposed as an adjunct to conventional caries prevention therapies ${ }^{(7)}$. Combing the effect of laser irradiation with re-mineralizing agent therapies, conducted that can be increase the resistance of tooth structure to mineral loss from the organic acids involved in dental caries ${ }^{(8,9)}$. Recently there are no studies addressing the combining the effect pulsed Nd;YAG laser and CPP-ACP with sodium fluoride paste on decreasing dental enamel de-mineralization, either the evaluation the effect of methylene blue stain of laser irradiating dental enamel surfaces. New approaches for caries prevention by using lasers effect combined re-mineralizing agent providing calcium and phosphate in addition to flouride for increasing the tooth mineral resistance needed,this study aimed to evaluate the effect of energy densities of pulsed (1064 nm) Nd:YAG laser on reducing human dental enamel de-mineralization, investigate the effect combing pulsed Nd:YAG laser with Casein-Phosphor-Peptide-Amorphous Calcium Phosphate containing $0.2 \%$ sodium Fluoride on reducing dental enamel, measurement of the temperature increase of the selected energy densities, measurement the Calcium atoms concentration in part per million (ppm) to determine the reduction of dental enamel demineralization, and measurement of the $(\mathrm{R}, \mathrm{G}, \mathrm{B})$ values as an indicator of the absorption of Methylene blue stain by demineralized dental enamel surfaces.

\section{MATERIAL AND METHODS}

Eighty sound extracted human first premolars extracted for orthodontic purposes $14-27$ years used in this vitro study. Sixty teeth were used for ions analysis and digital photographic colour value analysis .Sample surfaces were examined with the stereomicroscope X10 to check surfaces were free of detectable white spot lesions. The teeth were washed and debride of attached tissue by curette and ultrasonic. The teeth were then polished with fluoridefree prophylaxis paste. All prepared teeth were stored in deionized water with $0.1 \%$ thymol solution at $4 \mathrm{C}^{\circ}$. The solution was changed on weekly basis until the teeth were used.

The samples coated with nail varnish leaving circular window of $4 \mathrm{~mm}$ in diameter. Then they split into two halves mesio-distally and horizontally at the cemento-enamel junction by disc saw mounted on low speed handpiece under running distilled water.

\section{Samples irradiation}

Samples irradiated by two doses of pulsed Nd:YAG laser (Han's Laser system Pulsed Nd:YAG laser,PB 80, manufactured by Han's Laser Technology, Co.Shenzhen, China) ,60mJ (0.6 W), and $170 \mathrm{~mJ}(1.7 \mathrm{~W})$,pulse width $1 \mathrm{~ms}$, irradiated three times for three seconds with cooling interval $15 \mathrm{~s}$. Re-mineralizing paste was applied on the samples for three minutes and repeated four times, then exposed for laser irradiation and acid challenge by incubate them at controlled temperature of $37^{\circ} \mathrm{C}$ in $3 \mathrm{ml}$ of

Acetate-buffer solution $\mathrm{pH}$ value of 4.5 for 48 hours. The solution then analysed by an atomic absorption spectrophotometer in Ibn Sina .Co. Iraq.

\section{Thermal test}

The tooth was used in a hot water bath. The K-type of Basic accuracy $(0.05 \%$ rdg \pm 0.3 Celsius $)$ thermocouple was inserted into pulp chamber on the opposite site to the irradiated widow the thermal grease of thermal conductivity $>0.6 \mathrm{~W} / \mathrm{m}-\mathrm{k}$ injected inside the pulp chamber for better contact between the thermocouple and the dentine surface.

\section{Colour test}

The samples were immersed in $2 \%$ methylene blue solution for one minute.The access removed by gauze, the each sample photographed by using high Nikon camera (D3300,Tokyo,Japan) and computer image processing software Adobe Cs2 2014 on windows 8 the $(R, G, B)$ values were measured to assess the dental enamel colour changing.

\section{RESULTS}

The temperature test results for each sample of group $\mathrm{C}$ and $\mathrm{E}$ respectively analysed by descriptive statistics,and Student's t-test showing very high statistical significance group E over group C (Table 1)of $(p<0.05)$. Thicknesses measurements of both enamel and dentine measurement values for groups $C$ and $\mathrm{E}$ showing no statistical significance between the tested groups (Table2).

Atomic absorption analysis done by using ANOVA test that shows a very high significant difference $(p<0.0001)$ between and within tested groups for of calcium concentration in part per 
million (ppm) (Table 3). Calcium concentrations were decreased for lower laser does but the significant reduction in lower laser dose with MI plus paste.

Colour (R, G, B) values analysis measurements analysed by ANOVA test that shows a very high significant difference $(p<0.0001)$ for $(R, G, B)$ values for all samples of each group respectively (Figure1). Groups C and D are significantly different in absorbing methylene blue stain in comparison to other groups.

\section{DISCUSSION}

The temperature was measured for group $\mathrm{C}$ (60 $\mathrm{mj})$, and group $\mathrm{E}(170 \mathrm{mj})$ to insure that the used parameters were not harmful for the dental pulp tissue ${ }^{(13)}$. The light should be transformed into heat in an efficient manner without causing thermal damage to the adjacent or subjacent tissues, the maximum temperature inside the pulp recorded for $\mathrm{GC}$ and $\mathrm{GE}$ were $2.5 \mathrm{oC}$, and $4.7 \mathrm{oC}$ respectively, with maximum average enamel and dentine wall thicknesses measured $3 \mathrm{~mm}$ and $3.1 \mathrm{~mm}$ respectively. Both temperature records of GD and GE seems fall within the safety limits, so there is agreement with the study ${ }^{(14)}$. According to Beer-Lambert's law (the basic law of thermodynamics), which states that there is an inverse relation between the thickness of the matter and the temperature deposited on it: $\mathrm{dQ}=\mathrm{m} \mathrm{c}$ where (dQ) is the heat content, (m) represents tooth mass, (c) is the heat capacity and (dT) is the linear change in the temperature.A hole was made opposite the lasing area, so that the thermocouple inserted inside the pulp chamber was isolated by the tooth structure, and may not detect the heat dissipated to that medium. A thermal compound was used inside the pulp chamber to prevent a gap formation between the thermocouple and the dentin ${ }^{(15)}$.

The acetate acid present in this solution was one of the three main acids produced by cariogenic plaque and, therefore produce the required demineralization pattern ${ }^{(16)}$.

The lower Calcium concentrations observed in group $\mathrm{C}(60 \mathrm{~mJ})$, and group $\mathrm{D}(60 \mathrm{mj}$ with remineralizing paste), with reduction in $\left(\mathrm{Ca}^{+2}\right)$ in ppt. percentage of $(25.7 \%, 21.75 \%)$ for group $\mathrm{C}$ with respect to negative and positive controls group respectively, While group D shows reduction percentage of calcium atoms concentration (33.34\%, $29.8 \%$ ) as compared with negative and positive controls groups respectively. The reduction of Calcium dissolution is probably due to a reduction of permeability of enamel ${ }^{(17)}$, a lower penetration of acid inside the underneath layers, and prominent chemical and mineral content changes ${ }^{(18)}$,this is agree with the pervious study ${ }^{(19,20)}$. While the $\operatorname{GE}(170 \mathrm{mj})$ and GF(170mj with MI paste plus)they are not showing a significant reduction in calcium concentration compared to GC and GD this is may be due to the energy dose that used for (GE and GF) was high to producing enamel acid resistance.

$R, G, B$ values are the three basic component of digital image. the colour assessment widely used in dentistry $^{(21)}$, and measurement of RGB value newly introduced as a new method for demineralization detection $^{(22)}$.the using a digital camera is an alternative way to use a color meter, so it is easy and low coast technique $^{(23)}$. The analysis used in this experimental research to show if there are any deference of tested groups to absorb methylene blue stain, and hence indicate the effect of acid dissolution on dental enamel samples. The results show a highest R,G and $B$ values for GC and GD as compared to the groups A and $\mathrm{B}$. These high values indicate brighter color and therefore less permeability of enamel surfaces, which means less demineralization, so there is agreement with other proposal(24). However R,G,B values for groups $\mathrm{E}$ and $\mathrm{F}$ were not significantly different from control groups that indicating a higher absorption of methylene blue stain.Also this test results may agree with the atomic absorption test results for groups $\mathrm{C}$ and $\mathrm{D}$ that showing lesser calcium concentration dissolution, that means the less demineralized enamel surfaces are poorly absorb stain .

\section{CONCLUSION}

The pulsed Nd:YAG laser of(60 mj ,11j/cm2 ) combing with Casein- Phosphor-Peptide-Amorphous Calcium Phosphate with Fluoride decreasing dental enamel demineralization.

The temperature elevation during using pulsed (1064 nm)Nd:YAG laser at(60 mj and $170 \mathrm{mj}$ ) was safe to the dental pulp ,and the colour analysis test showed decrease in stain absorption of enamel surfaces treated with $(60 \mathrm{mj})$ combined with the remineralizing paste .

Table1: Student's t-test of thermal records

\begin{tabular}{|c|c|c|c|c|c|}
\hline Group & Means & S.D & t-test & P-value & Sig. \\
\hline C & 2.22 & 0.131 & 35.77 & 0.0001 & VHS \\
\hline E & 4.49 & 0.152 & & \\
\hline
\end{tabular}


Table2: Student's t-test for thickness measurement between (C) and (E) groups.

\begin{tabular}{c|c|}
\hline Group \\
\hline C \\
E
\end{tabular}

\begin{tabular}{|c|}
\hline Means \\
\hline 2.92 \\
2.94
\end{tabular}

\begin{tabular}{|c|}
\hline S.D \\
\hline 0.079 \\
\hline 0.117 \\
\hline
\end{tabular}

t-test

\begin{tabular}{|c|c|}
\hline P-value & Sig. \\
\hline 0.66 & NS \\
\hline
\end{tabular}

Table3: multiple comparisons indicate significant differences in level among the groups for (Ca) in ppt.

\begin{tabular}{|c|}
\hline GROUPS \\
\hline Group A \\
\hline Group B \\
\hline Group C \\
\hline Group D \\
Group E \\
\hline Group F \\
\hline
\end{tabular}

\begin{tabular}{|c|}
\hline Mean \pm S.D \\
\hline $108.85 \pm 11.28(\mathrm{a})$ \\
$103.36 \pm 8.58(\mathrm{a}, \mathrm{b})$ \\
$80.87 \pm 2.6(\mathrm{c})$ \\
$72.56 \pm 2.078(\mathrm{~d})$ \\
$96.7 \pm 2.1(\mathrm{~b}, \mathrm{e})$ \\
$89.45 \pm 3.6(\mathrm{e})$ \\
\hline
\end{tabular}

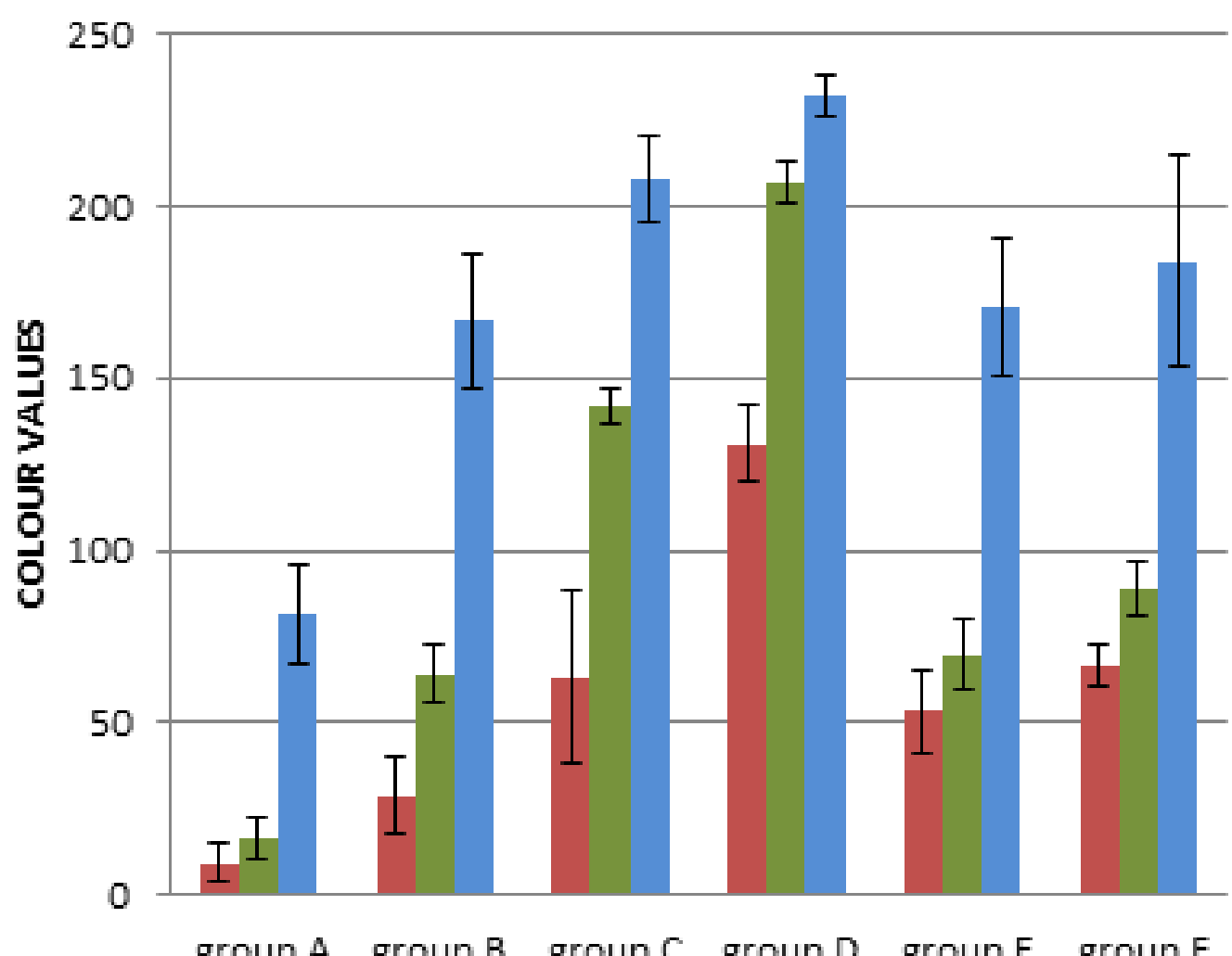

RED VALUES

GREEN VALUES

BLUE VALUES

group A group B group C group D group E group F

Figure1:( $R, G, B)$ values for all samples of each group

\section{REFERNCES}

1. Lima YBO, Cury JA. Seasonal variation of fluoride intake by children in a subtropical region. Caries Res. J 2003 ;37:335- 338 .

2. Shen P, Cai F, Nowiciki A, Vincent J, Reynolds EC. Remineralization of enamel sub surface lesions by sugar free chewing gum containing casein phosphopeptideamorphous calcium phosphate. Dent Res J.2001;80: 2066-70.

3. Reynolds EC. Remineralization of enamel subsurface lesions by casein phosphopeptide-stabilized calcium phosphate solutions. Dent Res.J 1997; 76(9):1587-95.

4. Reynolds EC, Cai F, Cochrane NJ, Shen P, Walker GD, Morgan MV, Reynolds C. Fluoride and casein phosphopeptide-amorphous calcium phosphate.Dent Res.J 2008 ;87(4):344-8.

5. Hamba H, Nikaido T, Inoue G, Sadr A, Tagami J: Effects of CPP-ACP with sodium fluoride on inhibition of bovine enamel demineralization: A quantitative assessment using micro-computed tomography. Dent J 2011; 39:405-413.

6. Stern, R.H., Sognnaes, R.F. \& Goodman F. Laser effect on in vitro permeability and solubility. Journal of the American Dental Association, 1966;7(3) 4:838-43.

7. Korytnicki D, Mayer MP, Daronch M, Singer Jda M, Grande rH. Effects of Nd:YAG laser on enamel microhardness and dental plaque composition: an in situ study. Photomed Laser Surg J 2006; 24:59-63.

8. Anderson J, Ellis R, Blankenau R, Beiraghi S, Westerman $\mathrm{G}$. Caries resistance in enamel by laser irradiation 
and topical fluoride treatment. J Clin Laser Med Surg (2000);18:33-36.

9. Westerman G, Flaitz CM Powell G, Hicks M, Enamel caries initiation and progression after argon laser irradiation: in vitro argon laser systems comparison. J Clin Laser Med Surg (2002); 20:257-262.

10. Featherstone JD, Nelson DG. Laser effects on dental hard tissues. Adv Dent J res 1987; 1:21-26.

11. Zach L, Cohen G. Pulp response to externally applied heat. Oral Surg Oral Med Oral Pathol J. 1965; 19:515-30.

12. Mohammed Abbood Al-maliky, Ali Shukur Mahmood, Christoph Kurzmann1, Markus Laky, Alexander Franz, Andreas Moritz, The effects of CO2 laser with or without nano-hydroxyapatite paste in the occlusion of dentinal tubules. Hidawi J 2014;798732: 5.

13. J.D. Featherstone and B.E. Rodgers,Effects of acetic, lactic and other organic acids on the formation of the artificial carious lesion Caries J Res.J 1981 ;15: 377.

14. Featherstone JD. Caries detection and prevention with laser energy. Dent Clin North AmJ 2000; 44:955-69.,141614.
15. Tagomori S, Iwase T. Ultrastructural change of enamel exposed to a normal pulsed Nd-YAG Laser. Caries ResJ 1995; 29:513-20.

16. Majori M, Manzon L, Pane S, Bedini r. Effects of Nd:YAG laser on dental enamel. J Appl Biomater Biomech J 2005; 3:128-133.

17. Bedini r, Manzon L, Fratto G, Pecci r. Microhardness and morphological changes induced by Nd:YAG laser on dental enamel: an in vitro study. Ann ist Super Sanita 2010; 46:168-172.

18. S.westland J,et al. Colour assessment in dentistry JAnnas of BMVA 2007;4:1-10.

19. Meller C, et al. Anew in vivo method for measuring caries activity with colour meter. J Clin Oral Investigation. 2006;10(2):140-144.

20. Zhao Shi-min, Wang Jun .De-/RE-minerlization in deciduous teeth assessed by color value analysis of digital photographs .Shanghai J Stomatology, 2011;20(5)527-530.

21. A.A.Kunin, etal. Coparative Analysis Of Effect Of Different Tooth Paste On Caries Resistance Of Dental Enamel .J. Clinical dentistry 2005;4. 